Proceedings of the 2011 Winter Simulation Conference

S. Jain, R. R. Creasey, J. Himmelspach, K. P. White, and M. Fu, eds.

\title{
INFORMED VIRTUAL GEOGRAPHIC ENVIRONMENTS: A GEOMETRICALLY PRECISE AND SEMANTICALLY ENRICHED MODEL FOR MULTI-AGENT GEO-SIMULATIONS
}

\author{
Mehdi Mekni \\ Institut National de Recherche Scientifique (INRS) \\ Eau Terre Environnement \\ 490, rue de la Couronne \\ Quebec (Quebec) G1K 9A9, CANADA \\ Bernard Moulin \\ Laval University \\ Department of Comp. Science \& Software Engineering \\ 1065, av. de la Medecine \\ Quebec,QC, G1V 0A6, CANADA
}

\begin{abstract}
In this paper, we propose a novel approach that extends our Informed Virtual Geographic Environment (IVGE) model in order to effectively manage knowledge about the environment and support agents' cognitive capabilities and spatial behaviours. Our approach relies on previous well established theories on human spatial behaviours and the way people apprehend the spatial characteristics of their surroundings in order to navigate and to interact with the physical world. It is also inspired by Gibson's work on affordances and knowledge provided by the environment to guide agent-environment interactions. The main contribution of our approach is to provide cognitive situated agents with: (1) knowledge about the environment represented using Conceptual Graphs (CG); (2) tools and mechanisms that allow them to acquire knowledge about the environment; and (3) the capability to reason about this knowledge and to autonomously make decisions and to act with respect to both their own and the virtual environment's characteristics.
\end{abstract}

\section{INTRODUCTION}

During the last decade, the Multi-Agent Geo-Simulation (MAGS) approach has attracted a growing interest from researchers and practitioners to simulate phenomena in a variety of domains including traffic simulation, crowd simulation, urban dynamics, and changes of land use and cover, to name a few (Benenson and Torrens 2004). Such approaches are used to study phenomena (i.e., car traffic, mobile robots, sensor deployment, crowd behaviors, etc.) involving a large number of simulated actors (implemented as software agents) of various kinds evolving in, and interacting with, an explicit description of the geographic environment called Virtual Geographic Environment (VGE).

A critical step towards the development of a MAGS is the creation of a VGE, using appropriate representations of the geographic space and of the objects contained in it, in order to efficiently support the agents' situated reasoning. Since a geographic environment may be complex and large scale, the creation 


\section{Mekni and Moulin}

of a VGE is difficult and needs large quantities of geometrical data originating from the environment characteristics (terrain elevation, location of objects and agents, etc.) as well as semantic information that qualifies space (building, road, park, etc.).

In order to yield realistic MAGSs, a VGE must precisely represent the geometrical information which corresponds to geographic features. It must also integrate several semantic notions about various geographic features. To this end, we propose to enrich the VGE data structure with semantic information that is associated with the geographic features. Moreover, we propose to abstract this semantically-enriched and geometrically-precise VGE description in order to enable large-scale and complex geographic environments modeling.

The rest of the paper is organized as follows: Section 1 provides a short survey of works on geographic environment representation as well as on agents' spatial behaviours. Section 2 briefly summarizes our IVGE computation model. Section 3 introduces the concept of Environment Knowledge (EK) and details the method that we propose to define it using Conceptual Graphs (CGs) (Sowa 1999). Section 4 provides a description of the proposed agent model and presents patterns of spatial behaviors. Section 5 illustrates, through a case study, how environment knowledge management supports situated agents's spatial behaviors in virtual urban environments.

\section{RELATED WORKS}

\subsection{Environment Representation}

Virtual environments and spatial representations have been used in several application domains. For example, Thalmann et al. proposed a virtual scene for virtual humans representing a part of a city for graphic animation purposes (Farenc, Boulic, and Thalmann 1999). Donikian et al. proposed a modeling system which is able to produce a multi-level data-base of virtual urban environments devoted to driving simulations (Thomas and Donikian 2003). More recently, Shao et al. proposed a virtual environment representing the New York City's Pennsylvania Train Station populated by autonomous virtual pedestrians in order to simulate the movement of people (Shao and Terzopoulos 2005). However, since the focus of these approaches is computer animation and virtual reality, the virtual environment usually plays the role of a simple background scene in which agents mainly deal with geometric characteristics. Indeed, the description of the virtual environment is often limited to the geometric level, though it should also contain topological and semantic information for other types of applications using advanced agent-based simulations. Current virtual environment models do not support large-scale and complex geographic environments and fail to capture real world physical environments' characteristics.

Not much research has been done on semantic integration in the description of a virtual environment. The Computer Animation and Behavioral Animation research fields provide a few attempts to integrate the semantic information in order to assist agents interacting with their environments. Semantic information has been used for different purposes, including the simulation of inhabited cities (Farenc, Boulic, and Thalmann 1999), computer animation (Kallmann 2001), and simulation of virtual humans (Garcia Rojas Martinez 2009). Farenc has first used the notion of Informed Environments (Farenc, Boulic, and Thalmann 1999). She defined informed environments as a database which represents urban environments with semantic information representing urban knowledge (Farenc, Boulic, and Thalmann 1999).

Despite the multiple designs and implementations of virtual environments, frameworks, and systems, the creation of geometrically-accurate and semantically-enriched geographic content is still an open issue. Indeed, research has focused almost exclusively on the geometric and topologic characteristics of the virtual geographic environment. However, the structure of the virtual environment description, the optimization of 


\section{Mekni and Moulin}

this description to support large-scale and complex geographic environments, the meaning of the geographic features contained in the environment as well as the ways to interact with them have received less attention.

\subsection{Spatial Behaviors and Knowledge Management}

Several theories in the field of human spatial behaviors have been proposed in order to explain how people navigate in the physical world, what people need to find their ways, and how people's visual abilities influence their decisions (Frank, Bittner, and Raubal 2001). Weisman identified four classes of environmental variables that influence spatial behaviours in physical worlds: visual access; architectural differentiation; signs to provide identification or directional information; and plan configuration (Weisman 1981). Seidel's study at the Dallas/Fort Worth Airport showed that the spatial structure of the physical environment has a strong influence on people's spatial behaviors (Seidel 1982). Information about the geographic environment along with the spatial and cognitive capabilities are fundamental inputs to the spatial decision-making process (Guo, Ren, and Wang 2008). These abilities are a necessary prerequisite to use environmental information or representations of spatial knowledge about the environment. This knowledge include information collected using perception capabilities, memorised information resulting from past experiences, and information provided by the environment it self (Jansen-Osmann and Heil 2007). The latter knowledge is known as "affordance".

The theory of affordances (Gibson 1979) is based on ecological psychology which advocates that knowing is a direct process: the perceptual system extracts invariants embodying the ecologically significant properties of the perceiver's world. Affordances have to be described relative to the person. For example, a chair's affordance "to sit" results from a bundle of attributes, such as "flat and hard surface" and "height", many of which are relative to the size of an individual. Many researchers believe that Gibson's theory is insufficient to explain perception because it neglects processes of cognition. In our approach, we suggest that exploiting the enriched description of virtual environments can provide agents with knowledge about their environment to support their spatial reasoning mechanisms and to help them make decisions that take into account the characteristics of their surroundings. Indeed, spatial agents require knowledge about their environment in order to reason about it, to infer facts, and to draw conclusions which will guide them to make decisions and to act. A number of challenges arise when creating knowledge about the environment for spatial agents' decision-making, among which we mention: 1) to represent knowledge using a standard formalism; 2) to provide agents with tools and mechanisms to allow them acquire knowledge about the environment; and 3 ) to infer and to draw conclusions and facts using premises that characterise the geographic environment.

\section{COMPUTATION OF IVGE}

In this section, we briefly present our automated approach to compute the IVGE data using vector GIS data. This approach is based on four stages: input data selection, spatial decomposition, maps unification, and finally the generation of the informed topologic graph (Mekni and Moulin 2007).

GIS Input Data Selection: The first step of our approach consists of selecting the different vector data sets which are used to build the IVGE. The input data can be organized into two categories. First, elevation layers contain geographical marks indicating absolute terrain elevations. Second, semantic layers are used to qualify various types of data in space. Each layer indicates the physical or virtual limits of a given set of features with identical semantics in the geographic environment, such as roads or buildings.

Spatial Decomposition: The second step consists of obtaining an exact spatial decomposition of the input data into cells. First, an elevation map is computed using the Constrained Delaunay Triangulation (CDT) technique. All the elevation points of the layers are injected into a 2D triangulation, the elevation being considered as an attribute of each node. Second, a merged semantics map is computed, corresponding to 


\section{Mekni and Moulin}

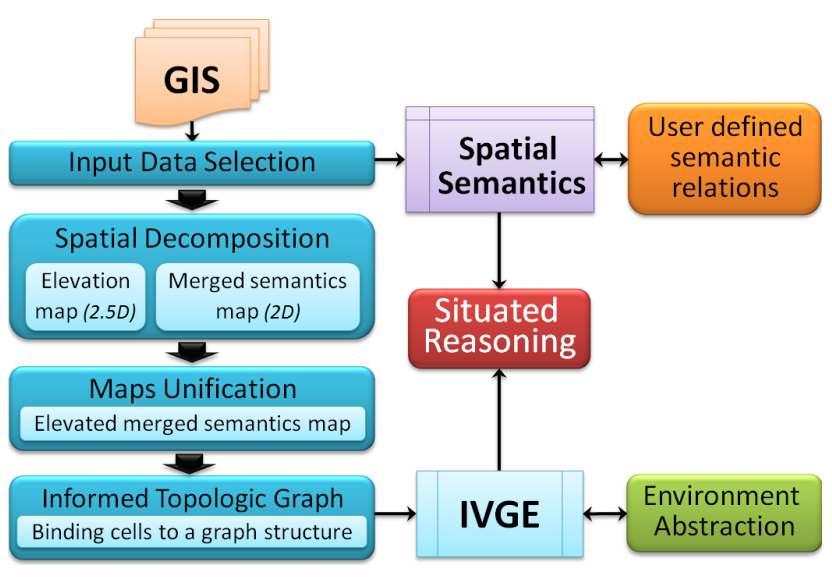

(a)

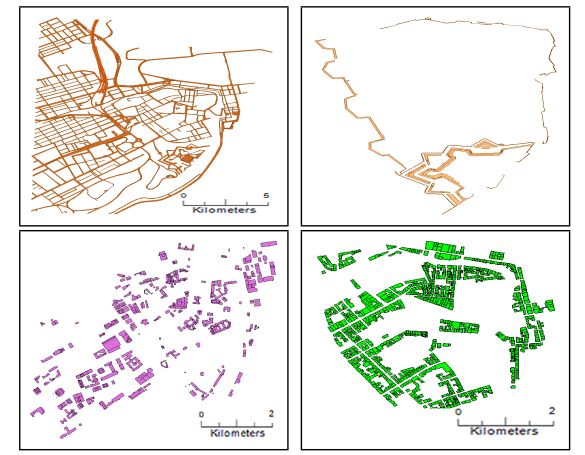

(b)

Figure 1: (a): topological graph representation of a CDT; (b): Various semantic layers related to Quebec City (Canada): road network; old city wall; governmental and private buildings.

a constrained triangulation of the semantic layers. Indeed, each segment of a semantic layer is injected as a constraint which keeps track of the original semantic data by using an additional attribute for each semantic layer.

Map Unification: The third step to obtain our IVGE consists of unifying the two maps previously obtained. This phase can be depicted as mapping the $2 D$ merged semantic map onto the $2.5 \mathrm{D}$ elevation map in order to obtain the final $2.5 \mathrm{D}$ elevated merged semantics map. First, preprocessing is carried out on the merged semantics map in order to preserve the elevation precision inside the unified map. Indeed, all the points of the elevation map are injected into the merged semantics triangulation, creating new triangles. Then, a second process elevates the merged semantics map.

Informed Topologic Graph: The resulting unified map now contains all the semantic information of the input layers, along with the elevation information. This map can be used as an Informed Topologic Graph (ITG), where each node corresponds to the map's triangles, and each arc corresponds to the adjacency relations between these triangles. Then, common graph algorithms can be applied to this topological graph, and graph traversal algorithms in particular.

\section{FROM SEMANTIC INFORMATION TO ENVIRONMENT KNOWLEDGE}

In (Mekni and Moulin 2010), we proposed a novel model along with a complete methodology for the automated generation of informed VGEs. Then, in we presented our abstraction approach which enriches and structures the description of the IVGE, using geometric, topologic and semantic characteristics of the geographic environment. In order to represent semantic information which characterises our informed virtual environment model, we also proposed to use Conceptual Graphs (CGs) (Sowa 1999). Our aim now is to evolve the semantic information to the level of knowledge and hence to build a knowledge-oriented virtual geographic environment in which spatial agents autonomously make informed decisions.

The process of making an informed decision has been modelled as a pyramid built on data (Lenat and Guha 1989) as shown on the left hand side of Figure 2(a). Data corresponds to the transactional, incremental physical records (Lenat and Guha 1989). In our IVGE model, this data corresponds to the geometric and geographic data provided by GIS. In and of itself this data is not sufficient to support spatial agents' decision-making. This data must be organized into information in order to be useful. Information is data that has been contextualized, categorized, often calculated (from initial data), corrected, 


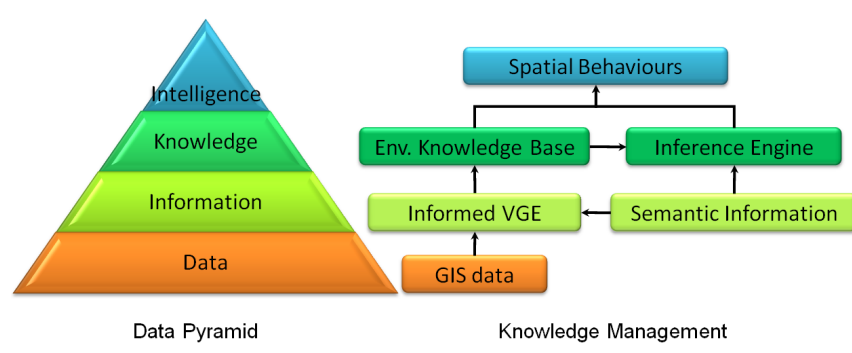

(a)

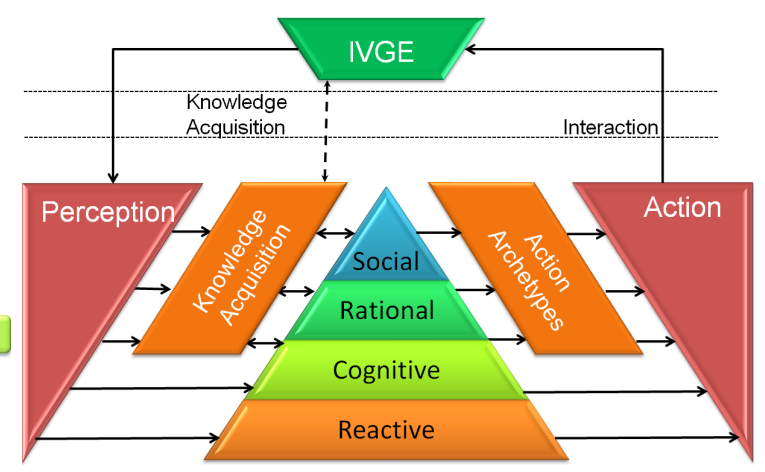

(b)

Figure 2: (a): The proposed knowledge management approach; on the left hand side, the pyramid data; on the right hand side, our knowledge management approach; (b): The enhanced perception-decision-action loop.

and usually condensed (Sajja 2008). In our IVGE model, information corresponds to the description of the IVGE resulting from the exact spatial decomposition of the geographic environment and enhanced with semantic information. Information often contains patterns within it and is sometimes useful for simple spatial behaviors such as motion planning. However, the context of these spatial behaviors can only be formed using some knowledge. Knowledge provides the next step of data organisation. For information to become knowledge, the context of the information needs to include predictive capabilities. Using predictive capabilities of knowledge, spatial agents can autonomously make informed decisions. The more complex and voluminous the underlying data sets are, the more effort is required to progressively organise it so that it becomes knowledge useful to the agents' decision-making. However, since our IVGE description is structured as a hierarchical topologic graph resulting from the geometric, topologic, and semantic abstraction processes, and since the semantic information is expressed using conceptual graphs, we are able to build knowledge about the environment to support agents' spatial behaviors.

\subsection{Environment Knowledge}

We define the notion of Environment Knowledge (EK) as "a specification of a conceptualization of the environment characteristics: the objects, agents, and other entities that are assumed to exist in the informed virtual geographic environment and the relationships that hold among them". Hence, EK is a description (like a formal specification of a program) of the spatial concepts (geographic features) and relationships (topologic, semantic) that may exist in a geographic environment. This description is provided by users in order to enrich the qualification of the geographic features which characterise the environment. It is expressed using a standard formalism which is close to natural language and computer tractable.

Let us emphasize that enhancing a multi-agent geo-simulation with EK, allows spatial agents to reason about the characteristics of the virtual geographic environment. Practically, EK is composed of spatial concepts (i.e., ask queries and make assertions) and spatial relationships (i.e., describe actions and behaviors). Our aim is to improve the perception-decision-action loop on which rely most agent models. Considering Newell's pyramid (Newell 1990) which comprises the reactive, cognitive, rational and social levels of agent behaviors, we mainly focus on the knowledge acquisition process in order to support the decision-making capabilities of spatial agents.

Conceptual graphs are widely used to represent knowledge (Sowa 1999). Actually, CGs enable us to formally represent spatial semantics characterizing our IVGE model and allow us to build a structured 


\section{Mekni and Moulin}

Environment Knowledge Base (EKB) based on a finite bipartite graph (Sowa 1999). The EKB allows MAGS users to represent, using a standard formalism, the information characterizing the virtual environment as well as the objects and agents it contains. Moreover, the EKB enables us to explicitly specify affordances (Gibson 1979) in order to support the agents spatial interactions with the informed virtual geographic environment in which they evolve.

To sum up, the EKB contains knowledge about the informed virtual geographic environment that an agent may use. This knowledge is provided basically by users to enrich the qualification of the geographic features which characterise the IVGE. Finally, this knowledge is structured using semantic concepts and relations expressed using conceptual graphs.

\subsection{Inference Engine}

Now that we have defined the environment knowledge base as a structure which contains explicit descriptions of geographic features using CGs, let us describe the Inference Engine (IE) which is part of our knowledge management approach. The IE is a computer program that derives answers from our environment knowledge base. Therefore, the IE must be able to logically manipulate symbolic CGs using formulas in the first-order predicate calculus. In order to acquire knowledge about the virtual environment, agents use the IE and formulate queries using a semantic specification that is compatible with CGs. Agents interpret the answers provided by the IE and act on the environment. They can also enrich the EKB by adding new facts that result from their observation of the virtual environment.

Conceptual graphs offer the opportunity to map knowledge about the environment into formulas in the first-order predicate calculus. We propose to use the Amine platform and Prolog+CG (Kabbaj 2006) in order to logically manipulate symbolic CGs and to provide spatial agents with an inference engine that allows them to query the environment knowledge, to acquire environment knowledge and reason about it.

In order to illustrate such a querying process, let us consider the following simple environment knowledge, composed of a set of two facts which provide an idea of the use of conceptual structures as a Prolog+CG data structure:

$\operatorname{cg}$ ([Man:Mehdi] $\leftarrow$ agnt-[Study]-loc $\rightarrow$ [University] $)$.

cg([Man:Mehdi] $\leftarrow$ agnt-[Play]-obj $\rightarrow[$ Soccer $])$.

And the following request: "Which actions are done by Man Mehdi?"

?- $\operatorname{cg}([$ Man:Mehdi $] \leftarrow$ agnt $-[x])$.

The answer provided by the Amine platform using its Prolog $+\mathrm{CG}$ inference engine is:

$x=$ Study;

$x=$ Play;

Now that we introduced the main parts of our environment knowledge management approach, namely EKB and IE, we detail in the following section the notions of agent and action archetypes and the way we use them to build spatial behaviors.

\section{FROM ENVIRONMENT KNOWLEDGE TO SPATIAL BEHAVIORS}

In order to characterize spatial agents, we propose to specify: (1) the agent archetype, its super-types and sub-types according to the semantic type hierarchy that we defined in 3 ; (2) the agent category (such as actor, object, and spatial area); and (3) the agent spatial behavioral capabilities, including moving within the IVGE content, perception of the IVGE and of other spatial agents. In the following subsections we discuss these elements. 


\section{Mekni and Moulin}

\subsection{Agent Archetypes}

In our environment knowledge management approach, the description of agents as well as objects and geographic features (spatial areas and zones) is enriched with semantic information. This means that these spatial agents belong to a semantic type hierarchy. Using the semantic type hierarchy allows us to take advantage of inheritance mechanisms. Hence, when modelling a MAGS involving a large number of agents, we only need to specify the attributes that are associated with the highest-level types of agents that we call agent archetypes rather than repeatedly specifying them for each lower-level agents. Let us define the Prolog+CG rule used to build a semantic type-hierarchy as follows: Supertype > Subtype1, Subtype2, ..., SubtypeN.

Entity $>$ Physical, Abstract.

Physical > Object, Process, Property.

Object $>$ Animate, Inanimate.

Animate $>$ Human, Animal, Plant.

Property > Juvenile, Adult, Gender, Yellow, White.

We now explain this example. The example starts at the top of the lattice with Entity. This super-type is then declared to have two immediate sub-types: Physical, and Abstract. The Abstract node is not associated with any subtype, and so remains a leaf node. The Physical node is given three immediate subtypes: Object, Process, Property, each of them being associated with subtypes. These subtypes may also have subtypes, and so on down the lattice.

Another important characteristic of agent archetypes is the 'multi-inheritance' property which allows an agent type to belong to two (or several) different agent archetypes and hence to inherit from their characteristics. Let us consider the following example.

Adult $>$ Woman, Man.

Young $>$ Girl, Boy.

Female $>$ Woman, Girl.

Male > Man, Boy.

Let us notice that Woman occurs at several places. This is allowed, as long as there is no circularity (i.e., as long as a type is not specified to be a subtype of itself) whether immediately or indirectly.

There is a fundamental difference between an archetype on the one hand, and instances of that type on the other hand. For example, while SchoolBus is an archetype, SchoolBusl and SchoolBus 2 are instances of that archetype. Instances are members of the group of entities which is named by the archetype. The archetype is the name of the group.

In Prolog $+\mathrm{CG}$, we have two ways of saying that a type has an instance: (1) we can simply declare it as an individual in the referent of some CG; (2) we can declare it at the top of the program in a catalog of individuals. A catalog of individuals for a given type is written as follows: Archeype = Instance1, Instance $2, \ldots$, InstanceN.

Here, a number of instances (on the right-hand side) are declared to be instances of a specific archetype (on the left-hand side). This rule is then repeated for each archetype that has instances. For example:

Entity $>$ Vehicle.

Vehicle $>$ Bus, Boat.

Bus > SchoolBus, CityBus.

SchoolBus = SchoolBus1, SchoolBus2. 


\section{Mekni and Moulin}

CityBus = CityBus1, CityBus2.

Boat $=$ OasisOfTheSeas, Titanic.

This example starts with a semantic type-hierarchy introducing the Entity, Vehicle, SchoolBus and CityBus agent archetypes (specified with $>$ ). This declares our archetypes. The SchoolBus archetype has two instances: SchoolBus1 and SchoolBus2. The CityBus archetype has two instances: CityBus1 and CityBus2. Also, the Boat archetype also has two instances: OasisOfTheSeas and Titanic.

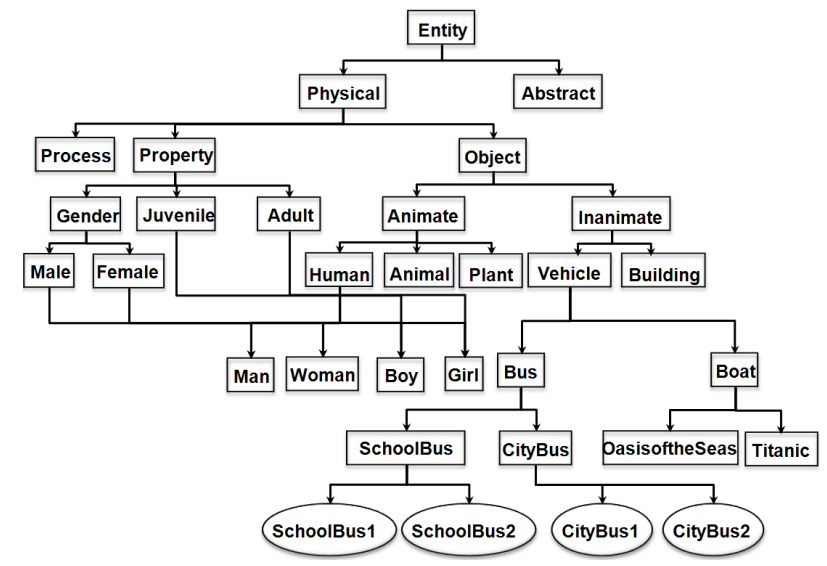

(a)

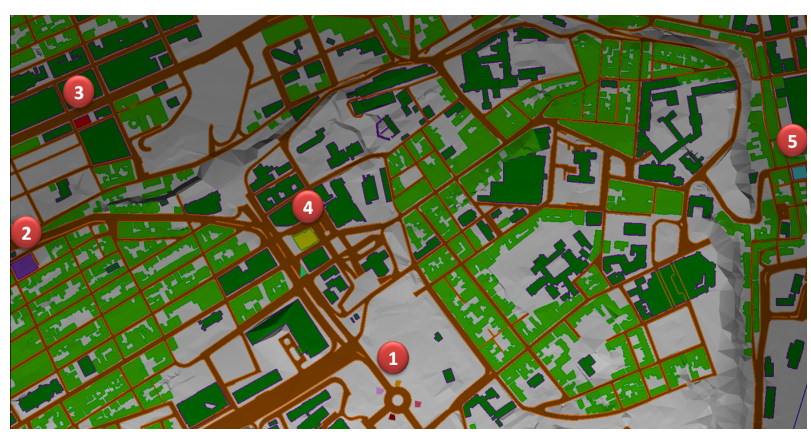

(b)

Figure 3: (a): A graph of Semantic Type Lattice with instances attached to agents archetypes (circle shapes); (b) The IVGE where the simulation takes place.

\subsection{Action Archetypes}

Since our research addresses the simulation of spatial behaviors, it has been influenced by some basic tenets of activity theory (Bellamy, Bødker, Christiansen, Engeström, Escalante, Holland, Kaptelinin, Kuutti, Nardi, Raeithel, Reeves, Velichkovksy, and Zinchenko 1996). In particular, our approach to manage environment knowledge rests on the commitments in activity theory that: (1) activities are directed toward objects, zones, or actors (Kaptelinin, Nardi, and Macaulay 1999); (2) activities are hierarchically structured; and (3) activities capture some context-dependence of the meaning of information (Mekni and Moulin 2010);

Theoretically, the common philosophy between our approach and activity theory is a view of the environment from the perspective of an agent interacting with it. Practically, we borrowed from activity theory two main ideas: (1) the semantics of activities and objects are inseparable (Kaptelinin, Nardi, and Macaulay 1999); and (2) activities, objects as well as agents are hierarchically structured (Bellamy et al. 1996).

We define an action archetype as a pattern of activities which are associated with agent archetypes. Hence, an action archetype describes a situation which involves one or several agent archetypes. We define an action archetype as a lattice of actions.

Let us consider the Vehicle agent archetype that we introduced in Section 4.1, page 7. Let us define the action archetype Drive that we associate with the Vehicle agent archetype as follows: an agent $* m$, which is a man, drives fast an object ${ }^{*} c$, which is a car.

This action archetype is expressed using the CG illustrated in Figure 4(a). And since the above description is equal or more specific than the action archetype presented in Figure 4(b). Then, it can be 


\section{Mekni and Moulin}

\author{
[DRIVE:*d] \\ $-($ agnt $) \rightarrow[$ MAN:*m] \\ $-(\mathrm{obj}) \rightarrow[$ CAR: $* c]$ \\ -(manr) $\rightarrow$ [FAST]
}

(a)

\begin{abstract}
[DRIVE:*d]
-(agnt) [HUMAN:*h]

$-($ obj $) \rightarrow$ [VEHICLE: *v]

$-(\mathrm{loc}) \rightarrow$ [NAVIGABLE]
\end{abstract}

(b)

\author{
[DRIVE:*d] \\ - (agnt) $\rightarrow[$ MAN:*m] \\ $-(\mathrm{obj}) \rightarrow\left[\mathrm{CAR}:{ }^{*} \mathrm{c}\right]$ \\ $-($ manr) $\rightarrow$ [FAST] \\ $-(\mathrm{loc}) \rightarrow$ [NAVIGABLE]
}

(c)
[DRIVE:*d]

-(agnt) $\rightarrow$ [STUDENT:*s]

$-(\mathrm{obj}) \rightarrow\left[\right.$ CAR: $\left.{ }^{*} \mathrm{c}\right]$

- (attr) $\rightarrow$ [YELLOW]

$-($ manr) $\rightarrow$ [FAST]

$-(\mathrm{loc}) \rightarrow$ [NAVIGABLE]

(d)

Figure 4: Various semantic layers related to Quebec City (Canada): (a) road network; (b) old city wall; (c) marina; (d) governmental buildings; (e) houses

inferred, by deduction, the CG detailed in Figure 4(c). And since Student is a sub-type of the archetype Man, the CG description illustrated in Figure $4(d)$ is also valid:

Hence, using the action archetype lattice and considering the fact that student is a subtype of man which itself is a subtype of human, we are able to deduce the following assumption: "an agent of type student drives fast an object of type car whose color is yellow". Using agent and action archetype hierarchies we are able to describe situations and infer by deduction a new ones.

\section{RESULTS}

In this section, we present a case study that illustrates how the IVGE model and the proposed knowledge management approach are used in practice. This case study aims to illustrate how spatial agents representing humans leverage the environment knowledge management approach that we propose. In order to acquire knowledge about the environment and to reason about it, spatial agents apprehend the virtual environment and make decisions according to their types and capabilities and taking into account its characteristics. In this example, a few human agents representing students and workers interact with the IVGE and our EKB in order to plan their path using a bus to get to their final destinations (university and office). This case study also involves a few agents of type CellAgent representing bus stations.

Let us consider three agent archetypes: Bus, Student, and Worker and several action archetypes including STOP, GO, GETIN, WALK and ROLL. The Bus archetype represents the different kinds of buses including city buses, school buses, etc. The Student and Worker archetypes includes respectively schoolchildness, pupils, students, and working persons. This case study involves an informed virtual geographic environment representing a part of Quebec City (Figure 3(b)). An environment knowledge base (EKB) is created using the Amine platform. In this EKB, we first specified the different semantic information that qualify our virtual urban environment. Second, we specified the above introduced agent archetypes namely, BUS, STUDENT, and WORKER. Two IVGE instances are specified: (1) HUMANNAV representing a view of the IVGE including the different geographic zones on which an agent of type human can move;(2) VEHICLENAV representing a view of the IVGE including the different geographic zones on which an agent of type vehicle can move. Besides, we specify the following facts: students and workers use buses to respectively reach universities and work places; humans walk on human navigable zones; vehicles roll on vehicle navigable zones; buses stop at stations.

$\operatorname{cg}([$ STUDENT] $\leftarrow$ agnt-[GETIN]-obj $\rightarrow$ [BUS] $)$.

$\operatorname{cg}([$ HUMAN] $\leftarrow$ agnt-[WALK]-loc $\rightarrow$ [HUMANNAV] $)$.

$\operatorname{cg}([$ STUDENT $] \leftarrow$ agnt-[GO]-loc $\rightarrow$ [UNIVERSITY] $)$.

$\operatorname{cg}$ ([VEHICLE] $\leftarrow$ agnt-[ROLL]-loc $\rightarrow$ [VEHICLENAV]).

$\operatorname{cg}([$ WORKER $] \leftarrow$ agnt-[GETIN] - obj $\rightarrow$ [BUS] $)$.

$\operatorname{cg}([$ WORKER $] \leftarrow$ agnt-[GO]-loc $\rightarrow$ [WORKPLACE] $)$.

$\operatorname{cg}([\mathrm{BUS}] \leftarrow$ agnt-[STOP]-loc $\rightarrow$ [STATION] $)$. 


\section{Mekni and Moulin}

In addition, two instances of buses, two instances of stations, and two instances of destinations are defined: Bus1, Bus2, Station1, Station2, $w$, and $u$. Bus1 which stops at station1 goes to the workplace $w$. Bus2 which stops at station2 goes to the university $u$.

$\operatorname{cg}([$ BUS: Bus1];-agnt-[GO]-loc $\rightarrow$ [WORKPLACE:w]).

cg([BUS: Bus2]i-agnt-[GO]-loc $\rightarrow$ [UNIVERSITY: u]).

$\operatorname{cg}([$ BUS: Bus1] $\leftarrow$ agnt-[Stop]-loc $\rightarrow$ [STATION: Station1]).

$\operatorname{cg}([$ BUS: Bus2] $\leftarrow$ agnt-[Stop]-loc $\rightarrow$ [STATION: Station2]).

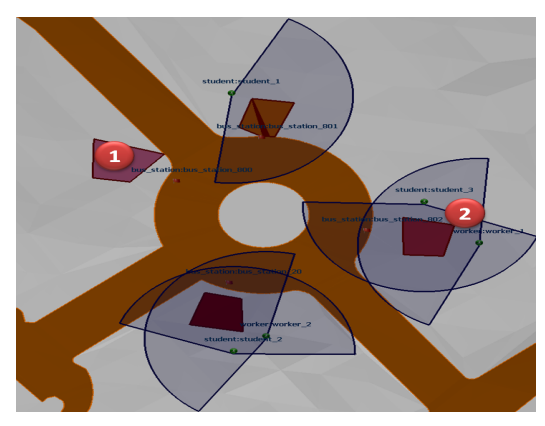

(a)

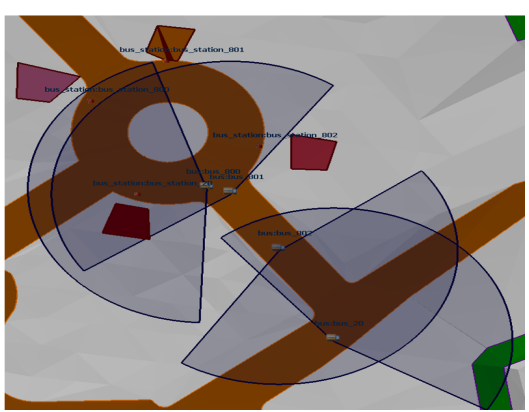

(b)

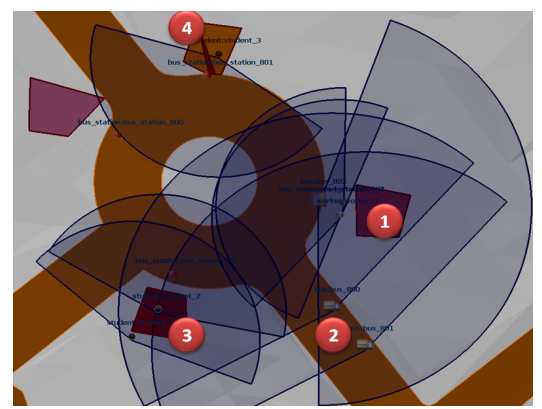

(c)

Figure 5: Stations, student and worker passengers, and buses: (a) (1) a group of cells representing a bus station of type CellAgent; (2) 3 students and 2 workers agents (green icon); (b) 4 agents of type Bus approaching the stations. Agents are associated with their respective perception fields which are highlighted in blue.

We carry out the simulation in which two agents of type student and three agents of type worker interact with the IVGE in which they evolve in order to localise the appropriate station from which they can catch the right bus to reach their final destinations. For simplification purposes, agents of type bus follow a pre-defined computed paths (Figure 5(b)). Agents of type student and worker start by identifying their own locations within the IVGE. Next, they interrogate the EKB in order to know which bus they should take in order to reach their final destinations (Figure 5(a)). The student agent asks the following query: "which bus goes to the university?"

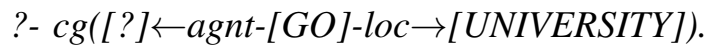

The answer provided by the Amine platform is:

$x=$ Bus 2 ;

Then, the student agent asks the following query:"where does Bus2 stop at?"

?- $\operatorname{cg}([B U S:$ Bus2] $\leftarrow$ agnt-[STOP]-loc $\rightarrow[?])$.

The answer provided by the Amine platform is:

$x=$ Station 2 ;

Once the answer is provided by the Amine platform, agents plan paths using this semantic description. Agents move towards the appropriate bus station, then wait for the bus. Since our agents are endowed with perception capabilities, they are able to detect when a bus arrives at the station. The agent bus is also endowed with the same spatial capabilities and waits at the station until all the agents get in it. 


\section{Mekni and Moulin}

\section{CONCLUSION}

In this chapter, we presented a knowledge management approach which aims to provide spatial agents with knowledge about the environment in order to support their autonomous decision making process. Our approach is influenced by some basic tenets of activity theory as well as by the notion of affordance. Our environment knowledge management approach is original in various aspects. First, a multi-agent geo-simulation model which integrates an informed virtual geographic environment populated with spatial agents capable of acquiring and reasoning about environment knowledge did not exist. Second, a formal representation of knowledge about the environment using CGs which leverages a semantically-enriched description of the virtual geographic environment has not yet been proposed. Third, providing agents with the capability to reason about a contextualised description of their virtual environment during the simulation is also an innovation that characterizes our approach.

Only a few research work have been found addressing the issue of spatio-temporal situations using standard formalisms. Among which, Haddad and Moulin's model (Haddad 2009) constitutes a potential improvement to our approach to support the qualitative analysis of the results of multi-agent geo-simulations. Coupling our environment knowledge management approach with Haddad and Moulin's qualitative model to analyze simulation results would provide an interesting knowledge-oriented multi-agent geo-simulation framework which would support: (1) the simulation of spatial behaviors using knowledge-based spatial agents; (2) the qualitative analysis and explanation of the simulation results; (3) the use of expertise by the agents' decision-making process; and (4) the agents self-learning from cases (i.e. case base reasoning). This is a promising avenue for our future works.

M. Mekni benefited from a PDF scholarship granted by FQRNT (Fonds Québécois de la Recherche sur la Nature et les Technologies). The author thank Dr Normand Bergeron, INRS, Qc and Dr Bernard Moulin, Laval University, Qc, for their advice during this research.

\section{References}

Bellamy, R., S. Bødker, E. Christiansen, Y. Engeström, V. Escalante, D. Holland, V. Kaptelinin, K. Kuutti, B. A. Nardi, A. Raeithel, J. Reeves, B. Velichkovksy, and V. P. Zinchenko. 1996, November. Context and Consciousness: Activity Theory and Human-Computer Interaction. First ed. The MIT Press.

Benenson, I., and P. Torrens. 2004. Geosimulation: Automata-Based Modeling of Urban Phenomena. John Wiley and Sons Inc.

Farenc, N., R. Boulic, and D. Thalmann. 1999. "An Informed Environment dedicated to the simulation of virtual humans in urban context". In Computer Graphics Forum (Eurographics '99), edited by P. Brunet and R. Scopigno, Volume 18(3), 309-318: The Eurographics Association and Blackwell Publishers.

Frank, A., S. Bittner, and M. Raubal. 2001. "Spatial and Cognitive Simulation with Multi-agent Systems". In Spatial Information Theory - Foundations of Geographic Information Science, Proceedings of COSIT 2001, Morro Bay, CA, USA, September 2001, edited by D. Montello, Volume 2205 of Lecture Notes in Computer Science, 124-139. Berlin, Heidelberg, New York: Springer.

Garcia Rojas Martinez, A. 2009. Semantics for Virtual Humans. Ph. D. thesis, École Polytechnique Fédérale de Lausanne.

Gibson, J. J. 1979. The Ecological Approach to Visual Perception. London: Lawrence Erlbaum Associates.

Guo, D., B. Ren, and C. Wang. 2008. Advances in Computation and Intelligence, Volume 5370/2008, Chapter Integrated Agent-Based Modeling with GIS for Large Scale Emergency Simulation, 618- 625.

Haddad, H. 2009. Une approche pour supporter l'analyse qualitative des suites d'actions dans un environnement géographique virtuel et dynamique: l'analyse "What-If" comme exemple. Ph. D. thesis, Department of Computer Science and Software Engineering, Laval University. 


\section{Mekni and Moulin}

Jansen-Osmann, P., and M. Heil. 2007. "The process of spatial knowledge acquisition in a square and a circular virtual environment”. Advances in Cognitive Psychology 3 (3): 389-397.

Kabbaj, A. 2006. "Development of Intelligent Systems and Multi-Agents Systems with Amine Platform". In ICCS'06: Proceedings of the 14th International Conference on Conceptual Structures, edited by H. Schärfe, P. Hitzler, and P. Øhrstrøm, Volume 4068 of Lecture Notes in Computer Science, 286-299: Springer.

Kallmann, M. 2001. Object Interaction in Real-Time Virtual Environments. Ph. D. thesis, École Polytechnique Fédérale de Lausanne.

Kaptelinin, V., B. Nardi, and C. Macaulay. 1999. "The activity checklist: a tool for representing the "space" of context". Interactions Magazine 6 (4): 27-39.

Lenat, D., and R. Guha. 1989. Building Large Knowledge-Based Systems; Representation and Inference in the Cyc Project. Boston, MA, USA: Addison-Wesley Longman Publishing Co., Inc.

Mekni, M., and B. Moulin. 2007, September. "Holonic Modelling of Large Scale Geographic Environments". In HOLOMAS'09: 4th International Conference on Industrial Applications of Holonic and Multi-Agent Systems. Linz, Austria.

Mekni, M., and B. Moulin. 2010, February 10-16,. "Hierarchical Path Planning for Multi-Agent Systems Situated in Informed Virtual Geographic Environments". In eKnow' 10: 2nd International Conference on Information, Process, and Knowledge Management, Volume 1, 48-55. Sint-Marteen, Netherlands Antilles: IEEE Computer Society.

Newell, A. 1990. Unified theories of cognition. Cambridge, Massachusetts: Harvard University Press.

Sajja, P. S. 2008. "Multi-Agent System for Knowledge-Based Access to Distributed Databases". Interdisciplinary Journal of Information, Knowledge, and Management 3:1-9.

Seidel, A. 1982. "Way-Finding in Public Spaces: The Dallas/Fort Worth, USA Airport". In Proceedings of the 20th International Congress of Applied Psychology. Edinburgh, Scotland.

Shao, W., and D. Terzopoulos. 2005. "Environmental Modeling for Autonomous Virtual Pedestrians". Digital Human Modeling for Design and Engineering Symposium.

Sowa, J. 1999, August. Knowledge Representation: Logical, Philosophical, and Computational Foundations. Course Technology.

Thomas, R., and S. Donikian. 2003.. "A model of hierarchical cognitive map and human memory designed for reactive and planned navigation". In Proceedings of the 4th International Space Syntax Symposium, Volume 1, 72-100. Londres.

Weisman, J. 1981, March. "Evaluating Architectural Legibility: Way-Finding in the Built Environment". Environment and Behavior 13 (2): 189-204.

\section{AUTHOR BIOGRAPHY}

MEHDI MEKNI Dr. Mehdi Mekni received his B.Eng. (2000) degree from the department of information sciences (University of Tunis - Tunisia), and his Ph.D. (2010) and M.Sc. (2006) degrees from the department of computer sciences at Laval University (Quebec City, Quebec, Canada). He is now a postdoctoral fellow at INRS. His main interests include multi-agent systems, agent-based geosimulation. His email address is mmekni@gmail.com.

BERNARD MOULIN obtained an Engineering degree from Ecole Centrale de Lyon (France), a Degree in Economics (University Lyon2) and a PhD (applied mathematics- computer science, University Lyon1). $\mathrm{He}$ is now a full professor at Laval University in the Computer Science Department. His main interests are: multi-agent systems, agent-based geosimulation, and application of AI techniques on modeling and simulation of software agents. bernard.moulin@ift.ulaval.ca. 